\title{
Savior in Desperate Situation: Successful TAVI for Critically Ill Patient with Severe Aortic Stenosis and Concomitant Constrictive Pericarditis Accompanied by Radiation Dermatitis, Complicated by Cold Abscess in Anterior Chest Wall
}

\author{
Hyungdon Kook, MD, PhD, Han Saem Jeong, MD, PhD, Cheol Woong Yu, MD, PhD \\ Division of Cardiology, Department of Internal Medicine, Korea University Anam Hospital, Korea University College of Medicine, \\ Seoul, Korea
}

\section{ABSTRACT}

Background: Constrictive pericarditis (CP) can coexist with severe aortic stenosis (AS), especially in patients with previous mediastinal radiation. Because impaired diastolic filling by $\mathrm{CP}$ may aggravate hemodynamic abnormalities from severe AS, leading to very low cardiac output, concomitant AS and CP result in a critical debilitating condition and pose a challenge to therapy.

Case Report: A 79-year-old woman was brought to our hospital with New York Heart Association class IV dyspnea and severe frailty (clinical frailty scale 8 ). She had a history of chronic constrictive pericarditis, severe aortic stenosis with reduced left ventricular systolic function (ejection fraction $40 \%$ ), paroxysmal atrial fibrillation, diabetes mellitus, and radiation dermatitis complicated by a cold abscess in the anterior chest wall from previous mediastinal radiation. She continually complained of dizziness, general weakness, and dyspnea despite optimal medical treatment, and her symptoms worsened recently while bedridden. Although simultaneous surgical pericardiectomy and aortic valve replacement is curative treatment, and the surgical risk was not high (Society of Thoracic Surgery score 4.745), her other comorbidities (radiation dermatitis, cold abscess, and severe frailty) eliminated the possibility of surgical treatment. Therefore, we decided on palliative treatment for $\mathrm{CP}$ after performing transcatheter aortic valve implantation (TAVI) for severe AS. We could not predict how she would recover from these conditions and were concerned about the high procedural risk associated with TAVI. Indeed, the patient had cardiac arrest during the TAVI procedure, and we implanted a 31-mm CoreValve while performing cardiac massage. After the patient recovered from cardiac arrest, we safely completed the TAVI procedure with a temporary pacemaker because of complete atrioventricular block. She recovered remarkably after TAVI with permanent pacemaker implantation, and is now able to walk without support.

Received February 27, 2020; received in revised form April 9, 2020; accepted April 9, 2020.

Correspondence: Cheol Woong Yu, MD, PbD, Cardiovascular Center (Interventional Cardiology), Anam Hospital, Korea University Medical Center. 126-1, Anam-dong 5ga, Seongbuk-gu, Seoul, 136-705, Republic of Korea; +82-10-7266-3389; fax: +82-2-927-1478 (e-mail: ycw717@naver.com).
Conclusion: Reduced diastolic filling by chronic CP aggravates hemodynamic deterioration through severe AS, leading to a very serious debilitating condition including severe frailty and decompensated heart failure. Although surgical pericardiectomy and aortic valve replacement are recommended as optimal therapy, TAVI alone can be an alternative therapeutic option if surgery is not possible.

\section{INTRODUCTION}

Constrictive pericarditis (CP) can coexist with severe aortic stenosis (AS), especially in patients with previous mediastinal radiation [Tsang 2015; Heidenreich 2009]. One of the main hemodynamic abnormalities observed in $\mathrm{CP}$ is reduced ventricular diastolic filling. $\mathrm{CP}$ can therefore lead to a reduction in stroke volume and cardiac output [Tsang 2015]. Because reduced stroke volume resulting from CP aggravates hemodynamic abnormalities from severe AS, leading to very low cardiac output, concomitant AS and CP result in a critical debilitating condition and pose a serious challenge to therapy [Tsang 2015]. We report successful transcatheter aortic valve implantation (TAVI) in a woman who recovered from severe frailty and a bedridden state resulting from decompensated heart failure, severe AS, and $\mathrm{CP}$ accompanied by radiation dermatitis, complicated by a cold abscess in the anterior chest wall.

\section{CASE REPORT}

A 79-year-old woman was brought to our hospital presenting with New York Heart Association (NYHA) class IV dyspnea and severe frailty (clinical frailty scale 8 ). Forty years earlier, she had undergone surgical mastectomy and received chemotherapy and mediastinal radiation therapy for breast cancer. She was first diagnosed with CP 8 years earlier after experiencing shortness of breath. Since that diagnosis, she had been on medication, but her symptoms had not improved. Her symptoms had begun to worsen 6 months earlier, and she complained of dyspnea, dizziness, and general weakness. She was diagnosed with several serious medical problems including chronic $\mathrm{CP}$, severe AS, atrial fibrillation, diabetes mellitus, and radiation dermatitis, complicated by a cold abscess in the anterior chest wall. 


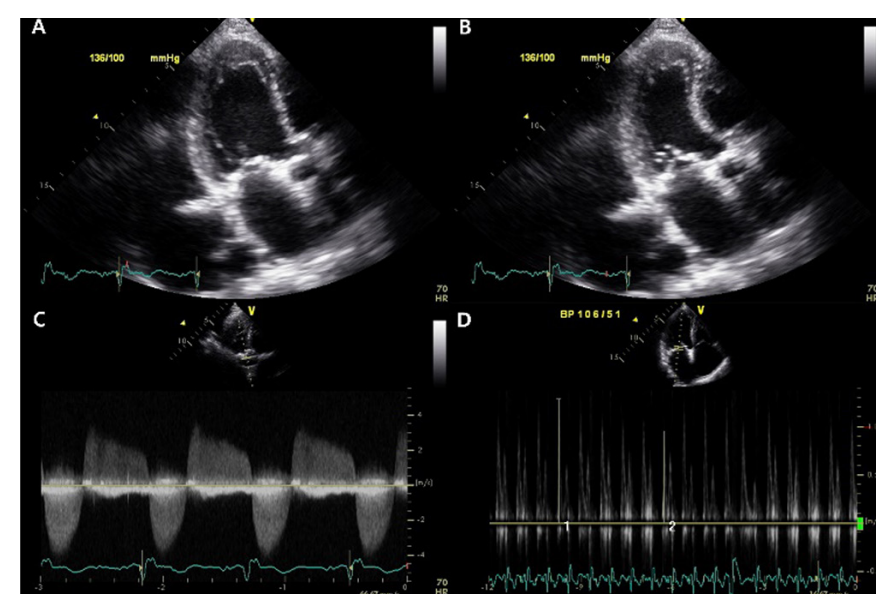

Figure 1. Transthoracic echocardiography showing constrictive physiology and severe aortic stenosis. A and B, Bouncing of interventricular septal wall motion in apical 4-chamber view. C, Elevated peak velocity of $4.2 \mathrm{~m} / \mathrm{s}$. D, Respiratory variation in early diastolic mitral inflow $(129 / 96 \mathrm{~cm} / \mathrm{s})$.

Echocardiography showed reduced left ventricular systolic function (ejection fraction $40 \%$ ) and hemodynamic parameters compatible with severe AS (aortic valve area [AVA] $0.59 \mathrm{~cm}^{2}$, peak/mean pressure gradient $70 / 42 \mathrm{mmHg}$, peak velocity $4.2 \mathrm{~m} / \mathrm{s}$ ), but a lower pressure gradient than expected for the grade of AS severity, plus CP physiology (Figure 1). Despite optimal medical therapy, her condition was aggravated, leading to severe debilitation.

We considered prescribing opiates to relieve the dyspnea; however, owing to concerns over hypotension and respiratory depression, opiates were not recommended for this patient. We determined that medication alone could no longer improve her condition. Because she had chronic $\mathrm{CP}$ and severe AS, surgical aortic valve replacement (SAVR) and pericardiectomy were needed for curative treatment. However, there were several obstacles to surgical treatment. First, she had radiation dermatitis complicated by a cold abscess in the anterior chest wall and radiation pleuritis (Figure 2), which had not healed with continuous conservative treatment. These problems made it impossible to perform sternotomy for open heart surgery. Second, she had severe frailty, which was associated with very high surgical risk. Accordingly, we considered TAVI to improve the patient's desperate situation by removing severe AS. We could not differentiate between severe frailty accompanied by severe AS or severe AS in the setting of chronic CP and several comorbidities; therefore, it was not possible to confirm whether TAVI could alleviate the patient's symptoms. In this clinical setting, however, TAVI was considered the only treatment option to have a chance. Therefore, the heart team concluded that palliative treatment after TAVI would be a much better option than curative treatment with surgical correction.

Because TAVI is associated with a high risk of potential complications, we made various preparations against the foreseeable complications. Initially, we tried to perform TAVI under conscious sedation out of a concern about a difficult recovery because of her lung problems, but the patient's vital

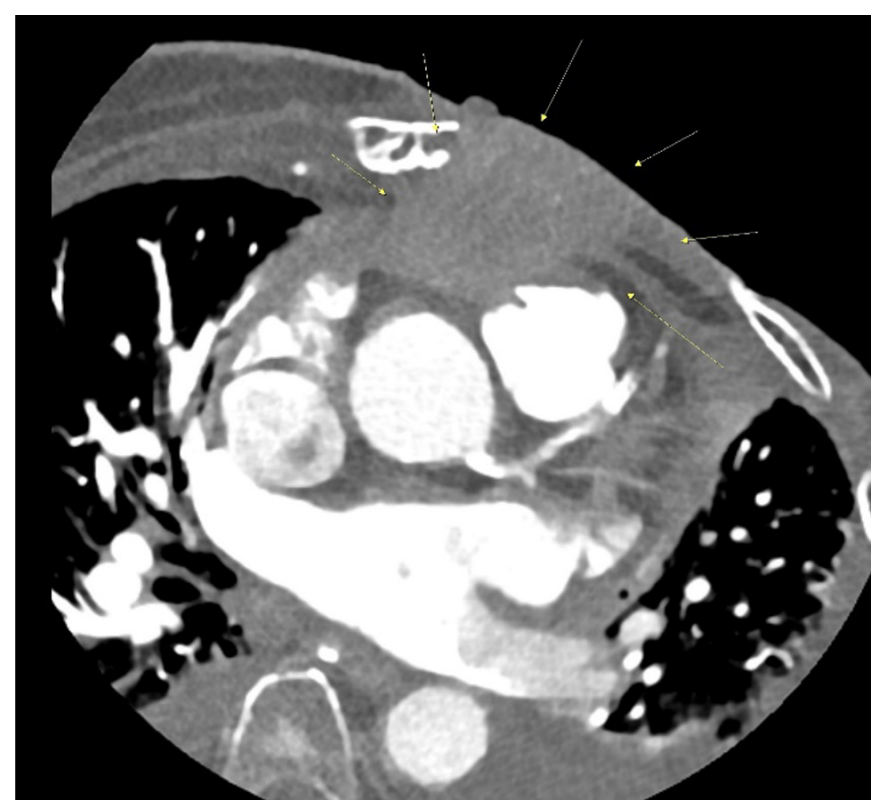

Figure 2. Chest computed tomography shows cold abscess in anterior chest wall (yellow arrow) and constrictive pericarditis.

signs became unstable. When we injected sedative drugs, her systolic blood pressure dropped below $60 \mathrm{mmHg}$ and was not restored even with rapid hydration and injection of dopamine and norepinephrine. Therefore, the original conscious sedation became general anesthesia with tracheal intubation.

A temporary pacemaker was introduced into the right ventricular apex via the right femoral vein. A 22-French TAVI sheath was introduced into the right common femoral artery. Pre-TAVI mean pressure gradient was $34 \mathrm{mmHg}$, which was lower than expected for the corresponding grade of AS severity given a slightly reduced left ventricular ejection fraction. During valve deployment, cardiac arrest occurred.

While performing cardiac massage with cardiopulmonary resuscitation, we rapidly deployed a $31-\mathrm{mm}$ CoreValve. The valve was successfully deployed with slightly lower apposition, and the patient was recovered from cardiac arrest (Figure 3). A transesophageal echocardiogram showed moderate paravalvular leakage (PVL); aortography showed moderate PVL and intact coronary arteries. The patient developed complete atrioventricular block and underwent temporary pacing at $70 \mathrm{bpm}$. To reduce the PVL, we performed postdilation with a $25-\mathrm{mm}$ balloon. During postdilation, ventricular tachycardia developed, and cardioversion was repeatedly performed to restore normal sinus rhythm. After postdilation, the PVL disappeared. The mean pressure gradient after TAVI was $8 \mathrm{mmHg}$. The patient underwent permanent pacemaker implantation because the complete atrioventricular block lasted for 7 days after TAVI.

The patient recovered from both heart failure and severe frailty after TAVI (from NYHA class IV to class I; from clinical frailty score 8 to 3). Echocardiography follow-up 1 year after TAVI showed improved ejection fraction of $57 \%$, effective orifice area of $1.8 \mathrm{~cm}^{2}$, effective orifice area index of $1.24 \mathrm{~cm}^{2} / \mathrm{m}^{2}$, 


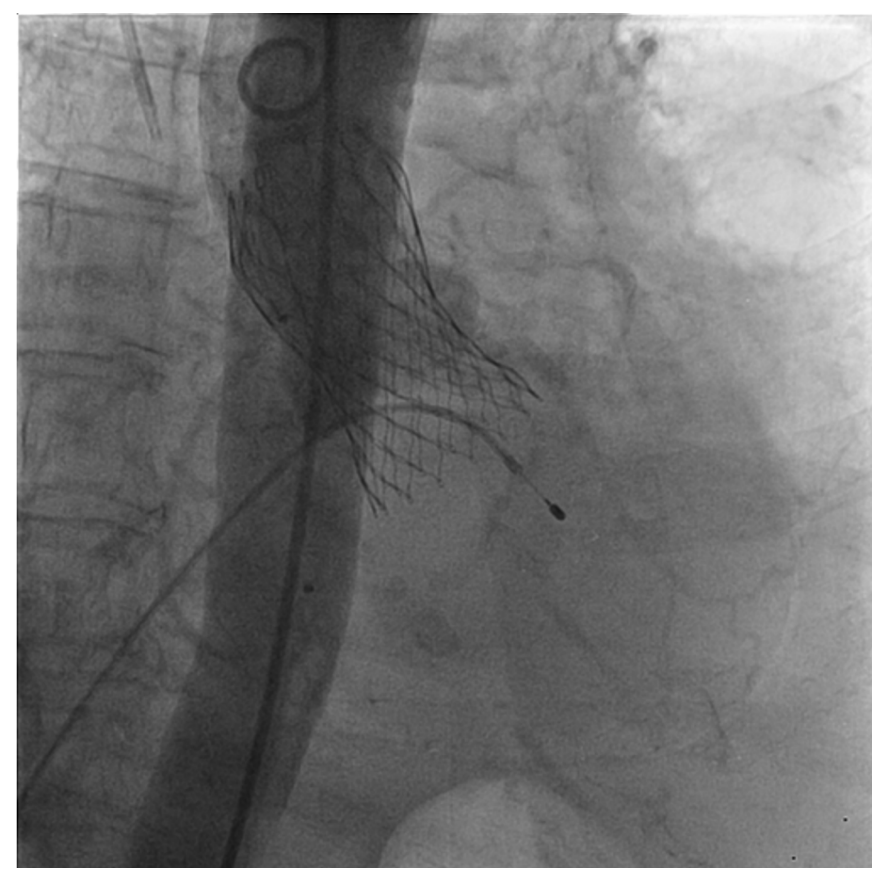

Figure 3. Aortogram after transcatheter aortic valve implantation.

mean pressure gradient of $4 \mathrm{mmHg}$, and peak velocity of 1.5 $\mathrm{m} / \mathrm{s}$. Right ventricular function was also improved in followup echocardiography. Respiration variation of mitral inflow and paradoxical ventricular septal wall motion with bouncing, which were consistently seen before TAVI, were decreased but still present. The patient was well managed (NYHA I to II) with optimal medical treatment of heart failure.

\section{DISCUSSION}

We report a successful TAVI procedure in a patient who recovered from a bedridden state due to decompensated heart failure and concomitant severe AS and CP accompanied by radiation dermatitis, complicated by a cold abscess in the anterior chest wall. Because the patient could not undergo SAVR and pericardiectomy, we decided to perform TAVI for severe AS and palliative therapy for CP. However, we could not determine whether AS or CP was the major contributor to the patient's critical condition and whether AS was the main cause of the patient's severe condition or simply a comorbidity. Thus we could not guarantee that TAVI alone would improve the patient's condition. Despite doubts about whether TAVI alone would be effective, there was no better choice.

CP leads to impaired ventricular diastolic filling and consequently reduced stroke volume and low cardiac output [Tsang 2015]. Because the transaortic gradient depends on stroke volume, it is expected to be lower for a given AVA in CP [Tsang 2015]. A study demonstrated that patients with concomitant AS and CP had disproportionately low transaortic gradients relative to the corresponding grade of AS and suggested that AS and CP were a potential mechanism for low-flow/low-gradient AS. Transaortic gradients are commonly reduced in the setting of CP despite preserved left ventricular ejection fraction, and this could be at least partially explained by a reduction in left ventricular stroke volume index [Tsang 2015]. Several studies have reported that regardless of ejection fraction, low-flow/low-gradient AS is associated with poorer prognosis than AS with normal flow and high gradient [Hachicha 2007].

Discordance between AVA and transaortic gradient has also been reported in studies in which $36 \%$ to $43 \%$ of patients with AVA $<1.0 \mathrm{~cm}^{2}$ had a mean transaortic gradient of $<40 \mathrm{mmHg}$, and reduced stroke volume was speculated to account for the low gradients [Pibarot 2012; Eleid 2013]. This discordance may have important implications for the management of AS. Low-flow/low-gradient AS with preserved ejection fraction in patients with combined AS and CP frequently causes underestimation of AS severity and leads to delay of treatment for severe AS and poor clinical outcomes. Therefore, a discordance of unknown cause must be investigated closely for hidden CP.

Curative treatment for severe AS and concomitant CP needs combined SAVR and pericardiectomy, which is very difficult because patients with AS and concomitant CP usually have high surgical risks [Tsang 2015]. Therefore, based on the surgical risk and magnitude of hemodynamic deterioration of each disease entity, surgeons must choose one of several options such as combined SAVR and pericardiectomy, SAVR alone, surgical pericardiectomy alone, or conservative therapy. Our patient had high surgical risk and several obstacles for open sternotomy, such as radiation dermatitis complicated by a cold abscess in the anterior chest wall. Therefore, we decided to perform palliative therapy for CP after TAVI for severe AS.

Surgical pericardiectomy is the only definitive treatment for chronic CP [Szabo 2013; George 2012]. Perioperative mortality of surgical pericardiectomy ranges from $6 \%$ to $7.1 \%$, and long-term survival after surgical pericardiectomy depends on etiology and patient characteristics. Patients who have chronic $\mathrm{CP}$ with idiopathic etiology have higher survival rates ( $\geq 80 \%$ at 5 to 7 years) than with those with chest irradiation etiology ( $0 \%$ to $30 \%$ at 5 to 10 years). The perioperative mortality rate of surgical pericardiectomy strongly correlates with the patient's NYHA class [Szabo 2013]. Our patient's etiology of chronic CP was mediastinal irradiation for breast cancer. Therefore, we decided on conservative treatment for $\mathrm{CP}$ based on the high operative risk and poor long-term survival after surgical pericardiectomy.

In this case, the definitive treatment was surgical pericardiectomy and aortic valve replacement. Even though the calculated surgical risk was not high, the patient had several other obstacles to surgery including the anterior chest wall abscess, radiation dermatitis, radiation pleuritis, and severe frailty. These problems made it impossible to perform a median sternotomy and for the patient to return to daily activities. Furthermore, the risk of surgical pericardiectomy is high, as described above.

Another discussion point of this case is whether TAVI alone may improve a patient's severe frailty and decompensated 
heart failure. We could not determine which-AS or $\mathrm{CP}-$ was the major contributor to patient's critical condition and whether the patient's severe condition was mainly from AS or was simply accompanied by AS. Thus we could not guarantee that TAVI alone would improve the patient's condition. Despite doubts about whether TAVI alone would be effective, there was no better choice. Fortunately, and surprisingly, the patient's conditions improved after TAVI.

Therefore, we concluded that TAVI alone may be a good alternative treatment to SAVR and pericardiectomy for patients with concomitant AS and CP if they have high surgical risk. However, the decision to perform TAVI as a part of a palliative concept has not been well established and should be approached with great care.

\section{REFERENCES}

Eleid MF, Sorajja P, Michelena HI, Malouf JF, Scott CG, Pellikka PA. Flow-gradient patterns in severe aortic stenosis with preserved ejection fraction: Clinical characteristics and predictors of survival. Circulation

\section{3;128:1781-1789.}

George TJ, Arnaoutakis GJ, Beaty CA, Kilic A, Baumgartner WA, Conte JV. Contemporary etiologies, risk factors, and outcomes after pericardiectomy. Ann Thorac Surg 2012;94:445-451.

Hachicha Z, Dumesnil JG, Bogaty P, Pibarot P. Paradoxical low-flow, low-gradient severe aortic stenosis despite preserved ejection fraction is associated with higher afterload and reduced survival. Circulation 2007;115:2856-2864.

Heidenreich PA, Kapoor JR. Radiation induced heart disease: Systemic disorders in heart disease. Heart 2009;95:252-258.

Pibarot P, Dumesnil JG. Low-flow, low-gradient aortic stenosis with normal and depressed left ventricular ejection fraction. J Am Coll Cardiol 2012;60:1845-1853.

Szabo G, Schmack B, Bulut C, et al. Constrictive pericarditis: Risks, aetiologies and outcomes after total pericardiectomy: 24 years of experience. Eur J Cardiothorac Surg 2013;44:1023-1028; discussion 1028.

Tsang MY, Choi JO, Borlaug BA, et al. Low-flow, low-gradient severe aortic stenosis in the setting of constrictive pericarditis: Clinical characteristics, echocardiographic features, and outcomes. Circ Cardiovasc Imaging 2015;8:e002812. 